\title{
Paraneoplastic cerebellar degeneration associated with somatic mutations in ultra-early diagnosis of small cell lung cancer: a case report
}

\author{
Xiao-Dan Shi", Yi Li", Ying He, Rui Wu, Fang Du, Gang Zhao \\ Department of Neurology, Xijing Hospital, the Fourth Military Medical University, Xi'an 710032, Shaanxi, China. \\ \#Authors contributed equally.
}

Correspondence to: Dr. Fang Du, Department of Neurology, Xijing Hospital, the Fourth Military Medical University, Xi'an 710032, Shaanxi, China. E-mail: dufang@fmmu.edu.cn; Dr. Gang Zhao, Department of Neurology, Xijing Hospital, the Fourth Military Medical University, Xi'an 710032, Shaanxi, China. E-mail: zhaogang@fmmu.edu.cn

How to cite this article: Shi XD, Li Y, He Y, Wu R, Du F, Zhao G. Paraneoplastic cerebellar degeneration associated with somatic mutations in ultra-early diagnosis of small cell lung cancer: a case report. Neuroimmunol Neuroinflammation 2020;7:68-72. http://dx.doi.org/10.20517/2347-8659.2019.16

Received: 16 Nov 2019 First Decision: 10 Jan 2020 Revised: 19 Jan 2020 Accepted: 3 Feb 2020 Published: 21 Mar 2020

Science Editor: Salvadeeswaran Meenakshi-Sundaram Copy Editor: Jing-Wen Zhang Production Editor: Tian Zhang

\begin{abstract}
Paraneoplastic cerebellar degeneration (PCD) can occur in patients with underlying cancer, such as small cell lung cancer (SCLC). Anti-CV2/CRMP5 antibodies are well-established biomarkers of PCD associated with SCLC, but cannot be detected in most situations. Recently, next-generation sequencing has been a promising technology to discover cancerdriven mutations, which provide an alternative strategy to accomplish ultra-early diagnosis of those patients. Here, we report the case of a 75-year-old man diagnosed with SCLC, who primarily presented with anti-CV2/CRMP5 antibodies positive PCD. Eight high-frequency gene mutations (TSC2, DNMT1, CIC, FGFG, NSD1, TSHR, CRLF2, and EPPK1) were detected 7 months before diagnosis with no abnormalities of imaging or cerebrospinal fluid examination found initially. Therefore, this case suggests the possibility of detecting certain somatic mutations for the ultra-early diagnosis of patients presenting with PCD associated with SCLC.
\end{abstract}

Keywords: Paraneoplastic cerebellar degeneration, small cell lung cancer, next-generation sequencing, ultra-early diagnosis

\section{INTRODUCTION}

As an autoimmune-mediated complication of cancer, paraneoplastic cerebellar degeneration (PCD) is associated with tumors such as lung cancer, gynecologic and breast cancers, and Hodgkin lymphoma ${ }^{[1]}$.

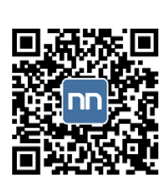


It is characterized by subacute onset gait instability that further develops into a pancerebellar syndrome with vertigo, diplopia, dysarthria, and truncal and appendicular ataxia ${ }^{[2]}$. Characterized by severe loss of cerebellar Purkinje cells, PCD is related to several antineuronal antibodies, such as anti-Yo, anti-Hu, and anti-CV2/CRMP5 antibodies ${ }^{[3]}$. Anti-CV2 antibodies, also known as anti-CRMP5 (collapsing responsemediator protein 5) antibodies, are well-established biomarkers of PCD associated with small cell lung cancer (SCLC), thymoma, and probably prostatic adenocarcinoma ${ }^{[4]}$. However, no antibodies are identified in approximately $40 \%$ of patients with a PCD due to unclear etiology of PCD or the limited detective technologies utilized ${ }^{[5,6]}$.

The cancer is primarily driven by the accumulation of somatic mutations in the genome, along with the contributions of epigenetic and transcriptomic alterations over one's lifetime. Revolutionary highthroughput DNA sequencing has become a promising and indispensable technique to study cancer ${ }^{[7]}$. On rare occasions, patient presents with a PCD months to years before cancer is diagnosed ${ }^{[8,9]}$. Therefore, whether the detection of certain mutations is available for clinical use in the ultra-early diagnosis of patients with PCD is worthy of further investigation.

This case highlights the fact that the detection of certain mutations by next-generation sequencing might plays a key role in ultra-early diagnosis of malignancy in a patient only displaying a PCD.

\section{CASE REPORT}

A 75-year-old man presented with a four-month history suggestive of progressive cerebellar symptoms in the form of nausea, vomiting, unsteadiness upon walking, unclear speech, and occasional choking when drinking water. He was treated in a local hospital. Cervical and cranial magnetic resonance imaging examinations and an electroencephalogram showed no obvious abnormalities. The cerebrospinal fluid (CSF) biochemical test showed a protein level of $0.5 \mathrm{~g} / \mathrm{L}$ (the rest is unknown) at this time.

He was admitted to our hospital on four occasions subsequently. Lumbar puncture was performed twice, and the results showed normal intracranial pressure. Cytology test results showed 6-11 white blood cells $/ \mathrm{mm}^{3}$, with a higher proportion of lymphocytes. Anti-CV2 antibodies were detected in both serum and CSF utilizing immunoblot techniques, but not immunofluorescence. The cancer-driven mutations were captured at the first visit by next-generation sequencing technology in CSF, and the results showed that eight genes (TSC2, DNMT1, CIC, FGF6, NSD1, TSHR, CRLF2, and EPPK1) had high-frequency mutations. Positron emission tomography-computed tomography scanning was performed in the third visit and no obvious lesions were seen in the parenchyma of the bilateral cerebellar hemisphere, with no signals of density or glucose metabolism changes in those areas.

Chest CT scanning was performed four times [Figure 1]. The first two scans only showed a right lower lobe inflammatory focus. The third scan showed old inflammation, along with enlargement of primary lymph nodes and the fourth scan performed on his fourth visit showed that the enlarged lymph nodes under the aortic arch were larger than before, and a shadow of soft tissue around the aortic arch of the left lower lobe probably reflected a malignant lesion. Histopathological evaluation of a biopsy specimen obtained from the posterior segment of the lung tissue revealed features of small cell cancer. Immunohistochemistry of the lung tissue was positive for CD117, CD56, CK8/18, Syn, and TTF-1 and negative for CgA, CK7, and P40, with a Ki-67 value-added index of $90 \%$. Therefore, morphological combined with immunohistochemical results supported the diagnosis of SCLC, seven months after the cancer-driven mutations were detected. The patient was given small doses of dexamethasone and rituximab $(100 \mathrm{mg})$, and the further treatment was administered at his local hospital's oncology department. 

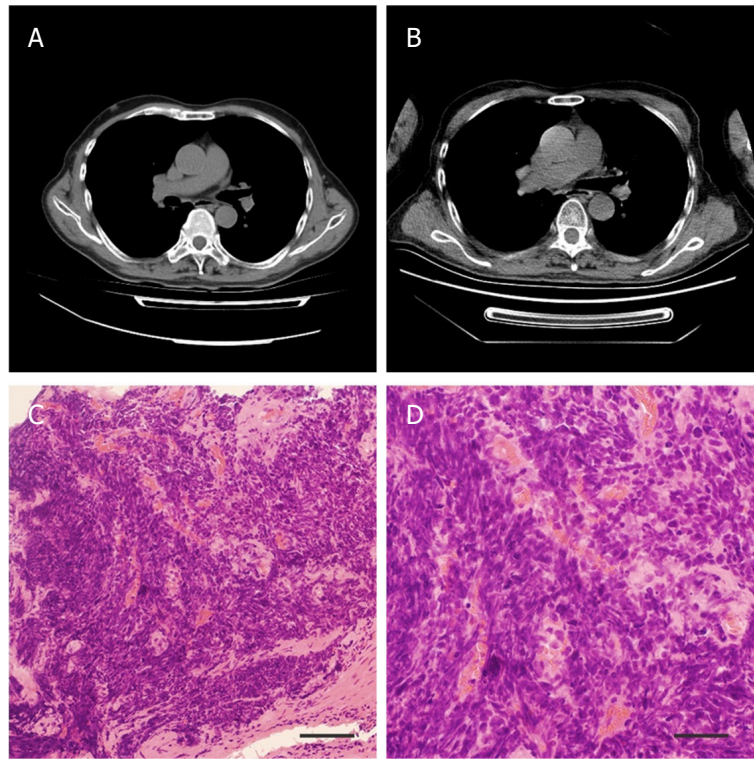

Figure 1. Chest $C T$ scans and pathological result. A: only a right lower lobe inflammatory focus can be found. The chest CT scan was performed at the same time the eight cancer-driven mutations were detected (May 2017); B: the enlarged lymph nodes under the aortic arch can be seen, and a shadow of soft tissue around the aortic arch of the left lower lobe probably reflects a malignant lesion (January 2018); C, D: morphological results reflect the possibility of small cell lung cancer. Scale bar: $400 \mu \mathrm{m}$ (C); and $200 \mu \mathrm{m}$ (D)

\section{DISCUSSION}

Given that PCD is associated with uncommon neuroendocrine tumors, such as SCLC, breast cancer, and prostate cancer, the possibility of malignancy in patients with a subacute cerebellar syndrome should be considered and excluded by clinicians. Several antineuronal antibodies are related to PCD, and more than $90 \%$ of patients harboring those antibodies are confirmed to have a tumor, mostly SCLC ${ }^{[10]}$. In patients with positive anti-CV2/CRMP5 antibodies, cerebellar ataxia, uveitis, and peripheral neuropathy are the most commonly occurring symptoms ${ }^{[6]}$. CRMP5, a protein belonging to a family of developmentally regulated neural proteins, expresses in regions of the central nervous system undergoing postnatal plasticity or in the peripheral nervous system mediating Schwann cell differentiation and axon repair ${ }^{[10]}$. In both anti$\mathrm{Hu}$ and anti-CV2/CRMP5 antibodies positive patients, SCLC was the most commonly found cancer, while malignant thymoma was found in patients only positive for anti-CV2 antibodies ${ }^{[11]}$. However, considering that the detection of those antibodies has very low sensitivity and specificity ${ }^{[6]}$, clinicians should be more vigilant, especially in cases with initial negative clinical examining results but with high suspicion of malignancy.

Together with epigenetic and transcriptomic alterations, cancer is primarily driven by the accumulation of somatic mutations in the genome over one's lifetime. In recent years, the identification of somatic mutations in cancer genomes has been revolutionized by high-throughput DNA sequencing and all types of somatic mutations can be revealed by whole-genome sequencing. In light of these advances, precision medicine and precision oncology have become possible, and treatments tailored based on an individual's mutational profile could be made.

In this case, no abnormalities were found via imaging or CSF examination until morphological and immunohistochemical results supported the diagnosis of SCLC, seven months after eight somatic mutations were detected. To be specific, we collected 278 somatic cancer-driven mutations from a cancer database and used target region sequencing to find candidate mutations on this occasion. Mutation frequency, variation prediction, and other analytic methods were utilized to analyze the data, and eight highfrequency gene mutations were identified in our patient: TSC2, DNMT1, CIC, FGF6, NSD1, TSHR, CRLF2, 
and EPPK1 (the method utilized here showed that the sensitivity, specificity, and diagnostic coincidence rate in diagnosing cancer of the central nervous system were $83.64 \%, 76.32 \%$, and $80.65 \%$, respectively, but the related article has not been published yet). Many of them have gained great attention in cancer studies. For example, TSC2 was reported to be related to sporadic pulmonary lymphangioleiomyomatosis ${ }^{[12]}$. The interaction between NSD1 and FLT3/ITD mutations determines the poor outcome of acute myeloid leukemia patients ${ }^{[13]}$. Aberrant expression of CRLF2, associated with mutated JAK2, was found to underlie the occurrence of acute lymphoblastic leukemia in Down syndrome ${ }^{[14]}$. CIC mutation is among the most studied of these genes, and has been well established to be tumorigenic in glioblastoma ${ }^{[15]}$. Hence, we hypothesize that the certain mutations found in this case may be related to SCLC with PCD, possibly mediated by the production of anti-CV2 antibodies.

Moreover, in this case, modest efficacy was initially achieved employing a treatment regimen utilizing a combination of dexamethasone and rituximab; however, there was no more significant clinical improvement achieved after the second treatment. A similar scenario was reported in a prior report about a patient presenting with PCD associated with squamous cell carcinoma of the tongue. A likely mechanism to account for this could be the irreversible neuronal tissue damage or the failure to normalize the cellular dysfunction caused by anti-CV2 antibodies in the long term ${ }^{[16]}$. Therefore, it calls for an alternative method of treatment for these patients targeting high anti-CV2 antibodies or their causative mutations.

Above all, our findings reveal certain mutations that might be related to PCD associated with SCLC to accomplish ultra-early diagnosis. To fulfill this goal, researchers need to overcome several challenges including analysis and interpretation of the sequencing data to understand the underlying mechanisms. Although this field is still in its infancy, pathogenesis, diagnosis, treatment, and prevention of cancer based on somatic mutations deserve more attention.

\section{DECLARATIONS}

\section{Acknowledgments}

We are grateful to Yining Yang (department of neurology, Xijing Hospital) for technical support and valuable comments on the manuscript.

\section{Authors' contributions}

Conception and design of the study: Zhao G, Du F

Performed data acquisition and provided technical and material support: Wu R, He Y

Performed data analysis and interpretation and drafted the work: Shi XD, Li Y

Read and approved the final manuscript: Shi XD, Li Y, He Y, Wu R, Du F, Zhao G

\section{Availability of data and materials}

The data and material could be available to readers upon request.

\section{Financial support and sponsorship}

The work was supported by National Key Research and Development Program of China (No. 2016YFC0904500) and Shanxi National Science Foundation (No. 2018kw-15).

\section{Conflicts of interest}

All authors declared that there are no conflicts of interest.

\section{Ethical approval and consent to participate}

Our research proposal has been reviewed and approved by the Independent Ethics Committee (I.E.C.), First Affiliated Hospital of Fourth Military Medical University on 19 May 2016. Number of ethics approval is No. KY20163367-1. The written informed consent was obtained from the patient. 


\section{Consent for publication}

Not applicable.

\section{Copyright}

(c) The Author(s) 2020.

\section{REFERENCES}

1. May ML, Dent S. Anti-Yo antibody-mediated paraneoplastic cerebellar degeneration associated with cognitive affective syndrome in a patient with breast cancer: a case report and literature review. Curr Oncol 2018;25:e585-91.

2. Mitoma H, Adhikari K, Aeschlimann D, Chattopadhyay P, Hadjivassiliou M, et al. Consensus paper: neuroimmune mechanisms of cerebellar ataxias. Cerebellum 2016;15:213-32.

3. Shams'ili S, Grefkens J, de Leeuw B, van den Bent M, Hooijkaas H, et al. Paraneoplastic cerebellar degeneration associated with antineuronal antibodies: analysis of 50 patients. Brain 2003;126:1409-18.

4. Aliprandi A, Terruzzi A, Rigamonti A, Bazzigaluppi E, Tremolizzo L, et al. Paraneoplastic cerebellar degeneration with anti-CV2/ CRMP5 antibodies and prostate adenocarcinoma. Neurol Sci 2015;36:1501-3.

5. Mason WP, Graus F, Lang B, Honnorat J, Delattre JY, et al. Small-cell lung cancer, paraneoplastic cerebellar degeneration and the Lambert-Eaton myasthenic syndrome. Brain 1997;120:1279-300.

6. Sabater L, Saiz A, Dalmau J, Graus F. Pitfalls in the detection of CV2 (CRMP5) antibodies. J Neuroimmunol 2016;290:80-3.

7. Levy SE, Boone BE. Next-generation sequencing strategies. Cold Spring Harb Perspect Med 2019;9.

8. Bhardwaj S, Khasani S, Benasher D, Stein EG, Meghal T, et al. Paraneoplastic cerebellar degeneration in nasopharyngeal carcinoma: a unique association. Cerebellum 2019;18:1126-9.

9. Abdulaziz ATA, Yu XQ, Zhang L, Jiang XY, Zhou D, et al. Paraneoplastic cerebellar degeneration associated with cerebellar hypermetabolism: case report. Medicine (Baltimore) 2018;97:e10717.

10. Antoine JC. Peripheral neuropathies associated with antibodies directed to intracellular neural antigens. Rev Neurol (Paris) 2014;170:570-6.

11. Antoine JC, Camdessanche JP. Paraneoplastic neuropathies. Curr Opin Neurol 2017;30:513-20.

12. Badri KR, Gao L, Hyjek E, Schuger N, Schuger L, et al. Exonic mutations of TSC2/TSC1 are common but not seen in all sporadic pulmonary lymphangioleiomyomatosis. Am J Respir Crit Care Med 2013;187:663-5.

13. Ostronoff F, Othus M, Gerbing RB, Loken MR, Raimondi SC, et al. NUP98/NSD1 and FLT3/ITD coexpression is more prevalent in younger AML patients and leads to induction failure: a COG and SWOG report. Blood 2014;124:2400-7.

14. Hertzberg L, Vendramini E, Ganmore I, Cazzaniga G, Schmitz M, et al. Down syndrome acute lymphoblastic leukemia, a highly heterogeneous disease in which aberrant expression of CRLF2 is associated with mutated JAK2: a report from the International BFM Study Group. Blood 2010;115:1006-17.

15. Bunda S, Heir P, Metcalf J, Li ASC, Agnihotri S, et al. CIC protein instability contributes to tumorigenesis in glioblastoma. Nat Commun 2019;10:661.

16. Saloustros E, Zaganas I, Mavridis M, Vamvakas L, Plaitakis A, et al. Anti-CV2 associated cerebellar degeneration after complete response to chemoradiation of head and neck carcinoma. J Neurooncol 2010;97:291-4. 